\title{
Assessment of gait stability, harmony, and symmetry in subjects with lower-limb amputation evaluated by trunk accelerations
}

\author{
Marco Iosa, PhD; ${ }^{1 *}$ Francesco Paradisi, MSc; ${ }^{1-2}$ Stefano Brunelli, MD; ${ }^{1}$ Anna Sofia Delussu, MD, PhD; ${ }^{1}$ \\ Roberto Pellegrini, CPO; ${ }^{3}$ Daniele Zenardi, CPO; ${ }^{3}$ Stefano Paolucci, MD; ${ }^{1}$ Marco Traballesi, MD $^{1}$ \\ ${ }^{1}$ Santa Lucia Foundation, Scientific Institute for Research, Hospitalization and Health Care, Rome, Italy; ${ }^{2}$ Department \\ of Human Movement and Sport Sciences, Interuniversity Centre of Bioengineering of the Human Neuromusculoskeletal \\ System, University of Rome "Foro Italico," Rome, Italy; ${ }^{3}$ ITOP SpA Officine Ortopediche, Rome, Italy
}

\begin{abstract}
Analysis of upper-body accelerations is a promising and simple technique for quantitatively assessing some general features of gait such as stability, harmony, and symmetry. Despite the growing literature on elderly healthy populations and neurological patients, few studies have used accelerometry to investigate these features in subjects with lower-limb amputation. We enrolled four groups of subjects: subjects with transfemoral amputation who walked with a locked knee prosthesis, subjects with transfemoral amputation who walked with an unlocked knee prosthesis, subjects with transtibial amputation, and age-matched nondisabled subjects. We found statistically significant differences for stability $(p<0.001)$, harmony $(p<$ $0.001)$, and symmetry $(p<0.001)$ of walking, with general trends following the noted order of subjects, but with the lowest laterolateral harmony in subjects with transtibial amputation. This study is the first to investigate upper-body acceleration of subjects with unilateral lower-limb amputation during walking who were evaluated upon dismissal from a rehabilitation hospital; it is also the first study to differentiate the sample in terms of level of amputation and type of prosthesis used.
\end{abstract}

Key words: accelerometry, ambulation, amputation, biomechanics, dynamic balance, gait, mobility, movement analysis, prosthesis, rehabilitation.

\section{INTRODUCTION}

Walking with a lower-limb prosthesis is a challenging task because missing a part of a limb alters the motor system, sensory feedback [1], and locomotor body schema [2]. This commonly implies slower and less efficient walking in subjects with amputation than in healthy subjects (HSs) [3-4]. Thus, walking speed (WS) and oxygen consumption have been widely used as indicators of gait ability and to assess rehabilitation outcomes or prosthetic components [5-8]. Conversely, little attention has been given to upright gait stability in the population with amputation $[1,4,9]$ and even less to gait harmony.

Upright gait stability has been defined as the capacity to minimize upper-body oscillations and absorb jerks, bumps, shakes, and fluctuations, despite the broad and fast movements of the lower limbs during locomotion [10-11]. Hence, an upright gait is stable when upperbody accelerations are minimized and smoothed, whereas

\footnotetext{
Abbreviations: $\mathrm{ANOVA}=$ analysis of variance, $\mathrm{AP}=$ anteroposterior, $\mathrm{BI}=$ Barthel Index, $\mathrm{CC}=$ craniocaudal, $\mathrm{HR}=$ harmonic ratio, $\mathrm{HS}=$ healthy subject, $\mathrm{L}=$ lumbar, $\mathrm{LK}=$ locked knee, LL = laterolateral, RM-ANOVA = repeated-measures analysis of variance, $\mathrm{RMS}=$ root-mean-square, $\mathrm{SRI}=$ symmetry ratio index, TFA $=$ transfermoral amputation, TTA $=$ transtibial amputation, UK = unlocked knee, WS = walking speed.

*Address all correspondence to Marco Iosa, PhD; Santa Lucia Foundation, Scientific Institute for Research, Hospitalization and Health Care, Via Ardeatina 306, 00179 Roma, Italy; +39-06-51501077; fax: +39-06-51501004.

Email: m.iosa@hsantalucia.it

http://dx.doi.org/10.1682/JRRD.2013.07.0162
} 
a distribution of accelerations in accordance with natural step-by-step repetition and bilateral evenness are signs of harmonic and symmetrical gait, respectively [12]. In the literature, the expression "gait stability" has been used with reference to the ability to walk with smoothed upper-body accelerations [13], as well as stride-to-stride kinematic variability [14] and local dynamic stability related to the ability of the locomotor system to maintain continuous motion by accommodating natural, infinitesimally small perturbations [15]. Although mathematically and conceptually different, all of these parameters have been found related to balance and risk of falling [14,16-17].

A large and growing body of literature has reported the successful use of accelerometers to investigate these gait features. In this study, we referred to gait stability as the ability to walk by smoothing upper-body accelerations [13], in accordance with many previous studies in HSs [18-22], patients with stroke [23-24], children with cerebral palsy [11-12], and people with lower-back pain [25] and cognitive impairments [26]. In people with amputation, upper-body accelerations during walking were investigated in three recent studies.

Tura et al. compared the upper-body accelerations of 10 subjects with transfemoral amputation (TFA) with those of 10 healthy controls during walking at low, natural, and fast speeds [9]. They found that a triaxial accelerometer (placed on the thorax at the xiphoid process) was adequate to assess gait features. They assessed symmetry and regularity of walking (using the autocorrelation coefficient of acceleration signals along the anteroposterior [AP] and craniocaudal [CC] axes, respectively), but not upright stability, as defined previously, or harmony of gait. In a more recent article, Tura et al. refined their analyses and identified the minimum number of strides (between 2 and 4) for a reliable computation of step symmetry based on accelerometric data [27].

Lamoth et al. not only focused their study on gait variability but also on upright gait stability in eight subjects with TFA compared with eight HSs during inside walking on smooth terrain, walking while performing a dual-task, and outside walking on even and uneven terrain [1]. Data were collected using a triaxial wearable accelerometer fixed with an elastic belt close to the center of mass at the level of lumbar (L) segment 3 .

These studies analyzed the walking of subjects with TFA by including subjects who had generally used a prosthesis for many years and were able to walk without aids [1,9]. It is conceivable that these subjects had already internalized into their locomotor body schema the mechanical characteristics of the prosthesis and the sensory feedback it provides at the residual-limb level and had developed efficient locomotor patterns as demonstrated by their WS higher than $1 \mathrm{~m} / \mathrm{s}$. Stability, harmony, and symmetry are conceivably more impaired in people with amputation after a rehabilitation period using a new prosthesis. Indeed, it can increase their risk of falling and reduce fluidity of movements [28].

The aim of our study was to assess upright gait stability, harmony, and symmetry in subjects with lowerlimb amputation upon dismissal from a rehabilitative hospital after receipt of prosthesis compared with a control group consisting of age-matched HSs. The parameters of these gait features were obtained by measuring upper-body accelerations in different types of amputation: subjects with transtibial amputation (TTA) and subjects with TFA who walked with an unlocked knee (UK) or locked knee (LK) prosthesis. Then, to identify which parameter was directly related to severity of the impairment, we assessed the correlation between the accelerometric parameters and clinical score obtained by the subject after rehabilitation for level of independence.

\section{METHODS}

\section{Participants}

Forty-four subjects were enrolled in this study: 22 with lower-limb amputation but no musculoskeletal or neurological comoborbities affecting gait not directly related to the amputation and 22 age-matched control HSs with no impairments. The amputation group included 14 subjects with TFA, 7 with an LK prosthesis (TFA-LK) and 7 with a UK prosthesis (TFA-UK), and 8 subjects with TTA. All were admitted to our rehabilitation hospital for a mean period of about $62 \mathrm{~d}$, with a mean of $45 \mathrm{~d}$ of prosthesis use. Receipt of prosthesis (for the first time or for a change of prosthesis) occurred on average $17 \mathrm{~d}$ after admission. Patients were assessed on 1 of the $3 \mathrm{~d}$ before dismissal from our rehabilitation hospital. The four groups of subjects (TFA-LK, TFA-UK, TTA, HS) were not statistically different in terms of age (analysis of variance [ANOVA]: $F=0.562, p=0.64$ ). Table 1 reports demographic and clinical data. The severity of impairment related to amputation was assessed in terms of the Barthel Index (BI); this is a clinical scale that evaluates independence in activities of daily living, ranging from 0 (completely dependent) to 100 (completely independent) [29]. 
Table 1.

Demographic and clinical features of four groups of enrolled subjects.

\begin{tabular}{|c|c|c|c|c|}
\hline Parameter & TFA-LK & TFA-UK & TTA & HS \\
\hline No. of Subjects & 7 & 7 & 8 & 22 \\
\hline Age, yr (mean \pm SD) & $65.0 \pm 5.8$ & $58.1 \pm 15.4$ & $56.9 \pm 5.3$ & $59.2 \pm 15.2$ \\
\hline \multicolumn{5}{|l|}{$\operatorname{Sex}(n)$} \\
\hline Male & 4 & 4 & 7 & 12 \\
\hline $\begin{array}{l}\text { Barthel Index Score } \\
\text { (mean } \pm \mathrm{SD} \text { ) }\end{array}$ & $87 \pm 8$ & $95 \pm 7$ & $99 \pm 1$ & $100 \pm 0$ \\
\hline Left & 6 & 7 & 2 & - \\
\hline Right & 1 & 0 & 6 & - \\
\hline \multicolumn{5}{|l|}{ Cause of Amputation ( $n$ ) } \\
\hline Vascular Disease & 4 & 3 & 5 & - \\
\hline Ischemic Event & 2 & - & - & - \\
\hline \multicolumn{5}{|l|}{ Aids Used $(n)$} \\
\hline 2 Crutches & 4 & 3 & 1 & - \\
\hline 2 Tetrapods & 1 & - & - & - \\
\hline No Aids & 2 & 4 & 7 & 22 \\
\hline
\end{tabular}

\section{Protocol}

The last 3 days before dismissal from our hospital, subjects performed the following assessment protocol: they performed the $10 \mathrm{~m}$ walking test commonly used in clinical settings [30]; then, they stood on a line marked on the floor and walked straight for $10 \mathrm{~m}$ at a selfselected speed until they arrived at another line on the floor in a $15 \mathrm{~m}$-long rehabilitation gym. During the test, they wore an elastic belt with a wearable inertial sensor device (FreeSense, Sensorize; Rome, Italy [sampling frequency $=100 \mathrm{~Hz}$, weight $=93 \mathrm{~g}]$ ) containing a triaxial accelerometer to measure accelerations along the three body axes (AP, laterolateral [LL], and CC) and three gyroscopes to measure angular velocities around the axes. Similar to Lamoth et al., the device was located on an area of the back corresponding to the L2-L3 spinous processes, close to the body center of mass [1]. During the test, subjects wore their commonly used shoes and subjects with amputation wore their prosthesis and used walking aids, if needed.

The time and number of steps needed to walk the $10 \mathrm{~m}$ were determined from the recorded peaks of AP acceleration [11-12]. In fact, foot strikes (which defined the beginning and the end of each step) were identified, in agreement with previous studies [12,24], as the moment at which a peak of AP acceleration occurred. Mean WS was computed as $10 \mathrm{~m}$ divided by the time spent to complete the test. Acceleration data of eight consecutive steps (four strides) performed in the central part of the walking pathway were analyzed. This approach has already been used and described in detail in previous studies in different populations of subjects $[12,24]$ and is in agreement with Tura et al.'s observation that when initial and final strides are excluded from the analysis, the minimum number of strides needed for reliable computation of step symmetry and stride regularity is about 2.2 and 3.5 strides, respectively, in subjects with amputation [27]. These signals were analyzed after their mean values were subtracted and after low-pass filtering at $20 \mathrm{~Hz}$ [12,19,31].

The following parameters were averaged for the four values of the four consecutive strides:

1. Root-mean-square (RMS) of acceleration, i.e., a measure of acceleration dispersion that coincides with the standard deviation because of signal mean subtraction [13].

2. Harmonic ratio (HR), i.e., the ratio between the sum of the amplitudes of the two main even harmonics and the 
sum of the amplitudes of the two main odd harmonics for the AP and CC axes or the reverse (odd/even) for the LL axis calculated via the discrete Fourier transform, which is an indicator of the rhythmicity between acceleration patterns and step repetition, with higher values corresponding to more harmonic gait [13].

3. Symmetry ratio index (SRI), i.e., the ratio between two consecutive minima of accelerations within each stride $\left(a_{\text {min }}\right)$, corresponding to the accelerations at foot strikes. This is a measure of the steep decelerations affecting the conservation of momentum. SRI was computed as the percentage ratio between two consecutive $a_{\min }$ relevant to two consecutive steps, each one performed by one leg (i.e., the ratio was computed between the lower $a_{\min }$ and the higher $a_{\min }$ absolute values). It provided values lower than 100 percent or equal to 100 percent (if the two consecutive steps had two equal values of $a_{\text {min }}$ ), representing an indicator of asymmetries in decelerations [12,32].

Because the RMS of acceleration is strictly dependent on WS, we normalized the values of RMS-AP and RMS-LL with respect to those of RMS-CC by using the inverse of their percentage ratio as an indicator of stability. This approach was shown to be suitable for the analysis of trunk accelerations of patients with stroke [24] based on the observation that CC accelerations were the least interesting from a locomotor control point of view [33]. In fact, Lamoth et al. also focused their study on AP and LL accelerations [1]. In detail, because RMS-AP/ RMS-CC and RMS-LL/RMS-CC are two normalized parameters related to instabilities [24], their inverses were used to quantitatively assess stability (Equations 1-2):

$$
\begin{aligned}
& \text { Upright stability: AP axis }=\frac{R M S-C C}{R M S-A P} \times 100 . \\
& \text { Upright stability: LL axis }=\frac{R M S-C C}{R M S-L L} \times 100 .
\end{aligned}
$$

Because it is conceivable that upright gait stability might be affected by alterations in prosthesis alignment, as is the case for postural standing stability [34], all prostheses were aligned by two highly experienced prosthetic technicians using L.A.S.A.R. Posture (Otto Bock HealthCare GmbH; Duderstadt, Germany), a system for the static alignment of prostheses that consists of a force- sensing platform and a projection system with laser and line optics.

\section{Statistical Analysis}

ANOVA was performed to assess differences in terms of age or comfortable WS in the four groups of subjects. Repeated-measures ANOVA (RM-ANOVA) was performed on acceleration RMS, normalized RMS, $\mathrm{HR}$, and SRI to analyze the main effect of group as a between-subject factor among the four groups, axis as a within-subject factor, and the effect of the interaction between group and axis. To take into account the possible effect of speed on the parameters extracted by acceleration signals, a similar RM-ANOVA was also performed including WS as a possible covariate variable [35]. Spearman correlation coefficient $(r)$ was computed between the BI score and each accelerometric computed parameter.

The threshold of significance was set at 0.05 for all analyses with the exception of post hoc comparisons, for which Bonferroni correction was applied on this threshold.

\section{RESULTS}

WS was found to be significantly lower in subjects with amputation than in HSs, especially for TFA-LK subjects (Table 2). Post hoc comparisons revealed that all three amputation groups walked significantly slower $(p<$ 0.001) than HSs. All comparisons among amputation groups were statistically significant $(p<0.009)$, except for between TFA-UK and TTA subjects $(p=0.69)$.

Because of these differences in speed, accelerations also resulted in significant differences among groups (Figure). When accelerations were normalized to take into account their strict relationship with velocity, LL and AP stability were found to be lower in subjects with amputation than in HSs. Post hoc comparisons revealed higher instability in TFA-LK subjects along the CC and AP axes with respect to TFA-UK subjects ( $p=0.01$ and $p=$ 0.02 , respectively) and along the CC axis with respect to TTA subjects $(p=0.01)$. No statistically significant differences were noted in amputation subgroups along the LL axis. The main factor group affected stability as well as its interaction with axis, indicating that LL stability was more affected in subjects with amputation than AP stability. 
Table 2.

Gait features for four groups of subjects.

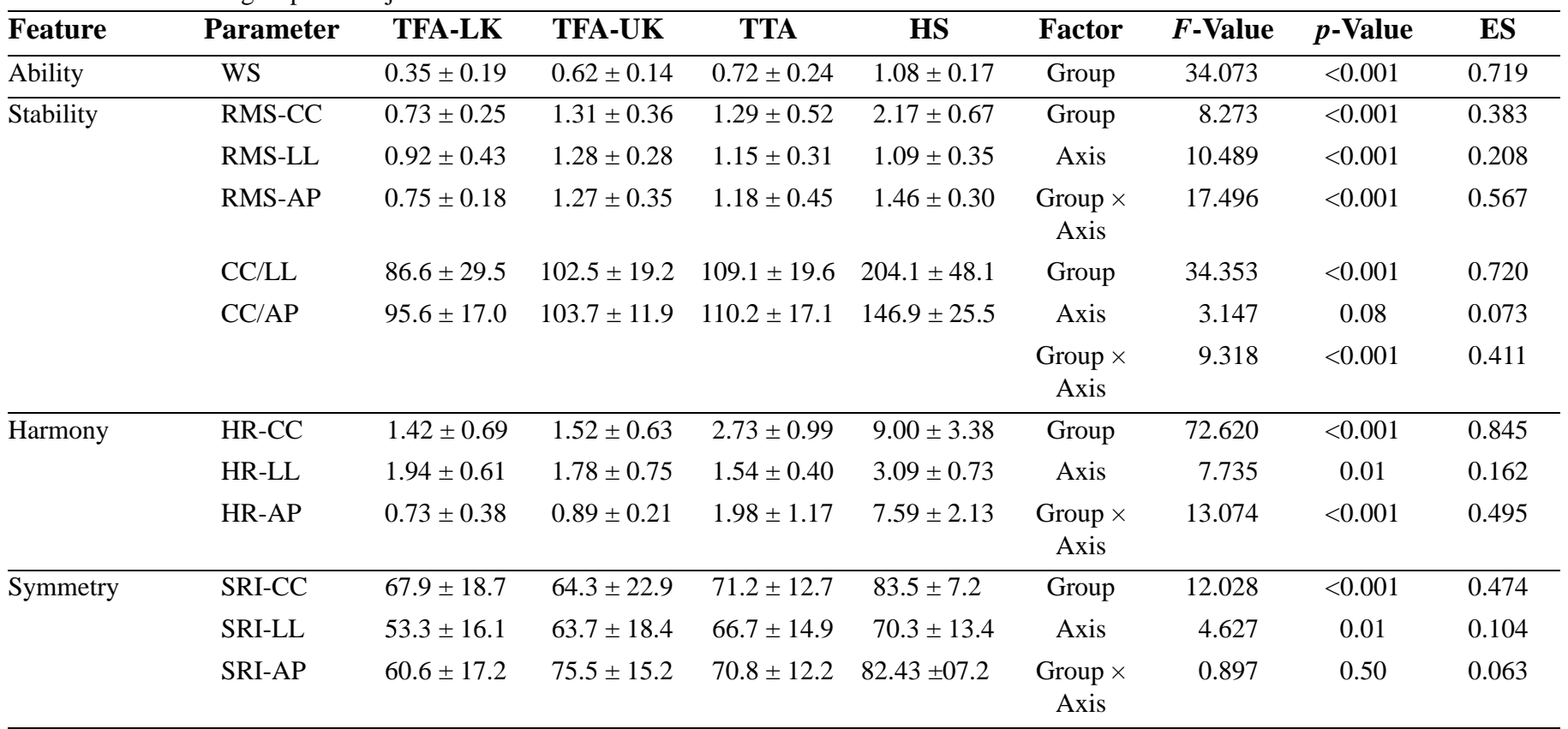

Note: Mean \pm standard deviation for computed values are reported. Results of ANOVA for WS and repeated-measures ANOVA for TFA-LK, TFA-UK, and TTA in terms of $F$-value, $p$-value, and ES (in terms of partial eta squared) are reported in last columns.

ANOVA $=$ analysis of variance, $\mathrm{AP}=$ anteroposterior, $\mathrm{CC}=$ craniocaudal, $\mathrm{ES}=$ effect size, $\mathrm{HR}=$ harmonic ratio, $\mathrm{HS}=$ healthy subject, $\mathrm{LL}=$ laterolateral, $\mathrm{RMS}=$ root-mean-square, SRI = symmetry ratio index, TFA-LK = subject with transfemoral amputation with prosthesis with locked knee, TFA-UK = subject with transfemoral amputation with prosthesis with unlocked knee, TTA = subject with transtibial amputation, WS = walking speed.

Significant differences were found among groups and among axes for harmony, which was also affected by the interaction of these two factors. Post hoc comparisons revealed that subjects with amputation had lower harmony than HSs $(p<0.001)$ along all three axes. Specific differences among amputation subgroups were found along the AP axis between TFA-LK and TTA subjects $(p=0.02)$ and along the CC axis between TFA-LK and TTA subjects ( $p=$ $0.01)$ and TFA-UK and TTA subjects $(p=0.02)$.

It should be noted that the general trend highlighted in the Figure for WS, RMS, normalized RMS, and HR following the order of impairment of TFA-LK, TFA-UK, and TTA subjects and HSs was not strictly respected by RMS-LL and especially not by HR-LL. This was mainly due to two subjects with TTA whose WS was $>1 \mathrm{~m} / \mathrm{s}$ (mean: $1.07 \mathrm{~m} / \mathrm{s}$ vs $0.61 \mathrm{~m} / \mathrm{s}$ in other TTA subjects) but whose mean HR-LL was lower than that of the other subjects (1.16 vs 1.67), despite higher HR-AP (3.51 vs 1.46 ) and SRI-LL (80.6\% vs 62.1\%).

Symmetry was significantly different among groups and axes but did not depend on the interaction group $x$ axis. Post hoc comparisons revealed that only TFA-LK subjects differed significantly from HSs along all three axes (AP: $p<0.001$, LL: $p=0.01$, CC: $p=0.01$ ). TFAUK subjects differed significantly from HSs only along CC $(p=0.01)$, and TTA subjects differed from HSs along CC $(p=0.01)$ and AP $(p=0.01)$ axes.

As reported in Table 2, the values of effect size for normalized RMS, HR, and SRI were higher for the factor group (about $0.7,0.8$, and 0.5 , respectively) than for axis or interaction between group and axis.

According to the approach suggested by Branch et al., analyses similar to those reported in Table 2 were also performed, with WS as a covariate variable [35]. With respect to the results reported in Table 2, the factor group continued to have a statistically significant effect on RMS $(p<0.001)$, normalized RMS $(p=0.01)$, and HR $(p<0.001)$ but not on SRI $(p=0.24)$. Furthermore, the interaction group $\times$ axis remained statistically significant for HR when WS was used as a covariate in the analyses.

Finally, the parameter values of the entire amputation group were found to be significantly correlated with BI score. Statistically significant correlations were found for 
WS $(r=0.676, p=0.01)$ and RMS-CC $(r=0.457, p=$ 0.03 ), and correlations close to statistical significance were also found for RMS-LL $(r=0.372, p=0.09)$ and RMS-AP ( $r=0.385, p=0.08)$. This was mainly due to the relationship between RMS and WS and, as expected, confirmed by the strict relationship between RMS-CC and WS ( $r=0.951, p<0.001$ ), which also supported its use in normalizing RMS-AP and RMS-LL. The BI score was also found to be significantly correlated with the sagittal harmony of walking HR-CC ( $r=0.661, p=0.01)$ and $\operatorname{HR}-\mathrm{AP}(r=0.604, p=0.01)$.

\section{DISCUSSION}

Our results showed greater instability and asymmetry in subjects with amputation than in HSs during walking. Similar to previous studies [1,9], we used a triaxial accelerometer to detect instability and asymmetry as well as loss of harmony. But differently from those studies, we divided subjects with amputation into three groups according to level of amputation and type of prosthesis. For most of the parameters investigated, we found a clear and significant trend related to the impairment (Figure). In fact, the lowest values of stability, harmony, and symmetry in walking were found for subjects with TFA who walked using a prosthesis with an LK compared with those who walked with a UK. The values computed for subjects with TTA were closer to physiological ones. The latter values, collected in a sample of HSs (age-matched with enrolled subjects with amputation), were in line with those already reported in the literature [1,24].

Regarding trunk accelerations, we found an important difference with respect to previous results: in our sample of subjects with amputation, trunk accelerations were lower than those of HSs; by contrast, Lamoth et al. found the reverse [1]. As already reported [24], trunk accelerations are strictly connected to speed and an increase in upper-body accelerations could be due to unsteady speed related to pathological instabilities as well as to an increase in WS related to better locomotor ability. In Lamoth et al. [1], the difference in speed between HSs and subjects with amputation during indoor level walking was only about 10 percent, which allowed for a direct comparison of accelerations. At similar velocities, higher trunk accelerations are a sign of instability. In our study, the difference in speeds was between 34 percent for TTA subjects and 68 percent for TFA-LK subjects with respect to HSs, implying the need to normalize accelerations. In fact, unlike subjects in previous studies [1,9], 20 out of 22 subjects with amputation enrolled in our study walked at a speed lower than $1 \mathrm{~m} / \mathrm{s}$. There were two main reasons for this: our sample included subjects with amputation being dismissed from a rehabilitative hospital after delivery of a first or a new prosthesis and they were 10 to $20 \mathrm{yr}$ older than those enrolled in the studies cited previously. On the other hand, our control subjects were also older than those enrolled in previous studies $[1,9,27]$ because they were age-matched with our sample of subjects with amputation. Nevertheless, in subjects with amputation, older age has been shown to be a negative prognostic factor affecting walking recovery after amputation [36]; indeed, it is probably stronger than the effect of aging on walking stability in HSs. It has also been suggested that reduced speed in severely impaired subjects may be a motor strategy to increase gait stability [37]. In fact, it was found that in subjects with amputation, the selfselected comfortable WS was lower than the most efficient one (whereas in HSs the two speeds coincided) [3]. It is conceivable that subjects with amputation prefer slow walking to increase stability and not to optimize gait efficiency. This is supported by the fact that the only two subjects with amputation who walked faster than $1 \mathrm{~m} / \mathrm{s}$ showed low harmony of gait along the LL axis. Less severely affected subjects with TTA probably use a compensation strategy to increase the speed and functionality of AP movements, based on LL compensation, indicating loss of stability and harmony. This could be why higher accelerations were found along the LL axis for TFA-UK and TTA subjects than HSs: the higher locomotor ability of TTA subjects (walking without aids and faster than TFA subjects) contributed to the LL harmony of walking. On the other hand, Lamoth et al. found that LL stability was most affected when the task difficulty was progressively increased, such as during walking on uneven terrain [1]. The trend among groups was found for parameters evaluated along the AP and CC axes, i.e., on the sagittal plane, but this trend, as well as correlation with the BI clinical score, was lost on the LL axis. This suggests that the LL accelerations of subjects with amputation do not depend directly on level of amputation, type of prosthesis, or clinical impairment (BI score). HSs are able to self-select a speed that optimizes efficiency, comfort [3], and harmony [13] of walking. Probably at the end of the rehabilitation program, subjects with amputation should choose a speed and try to optimize one or more 


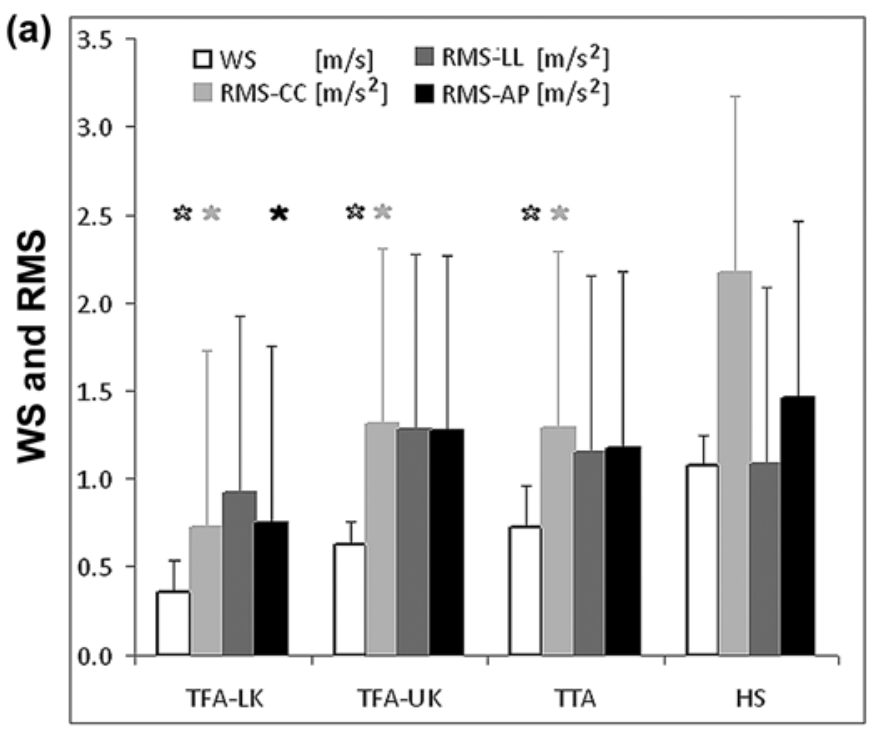

Subjects

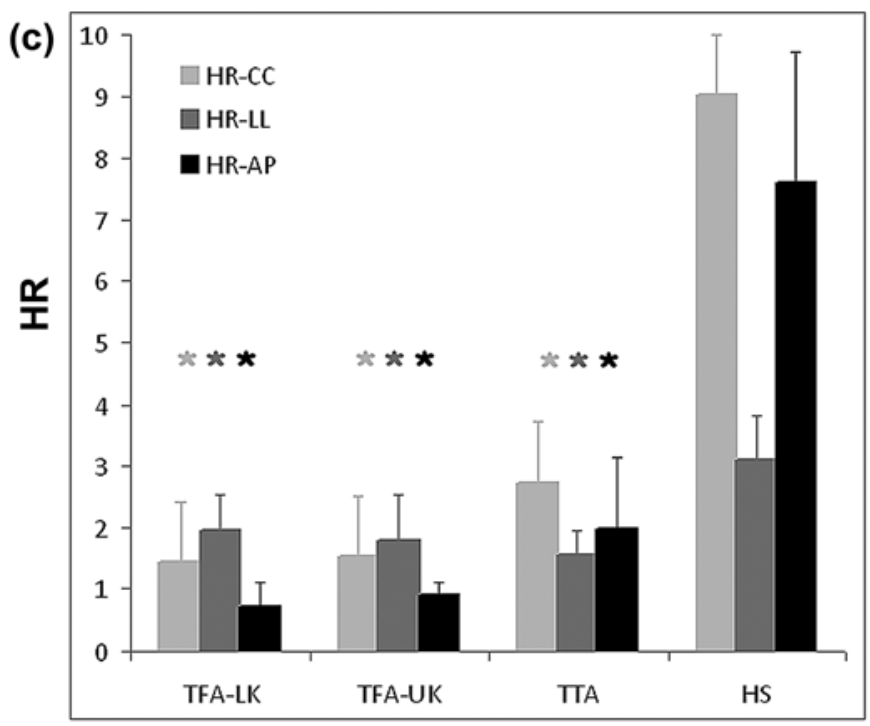

Subjects

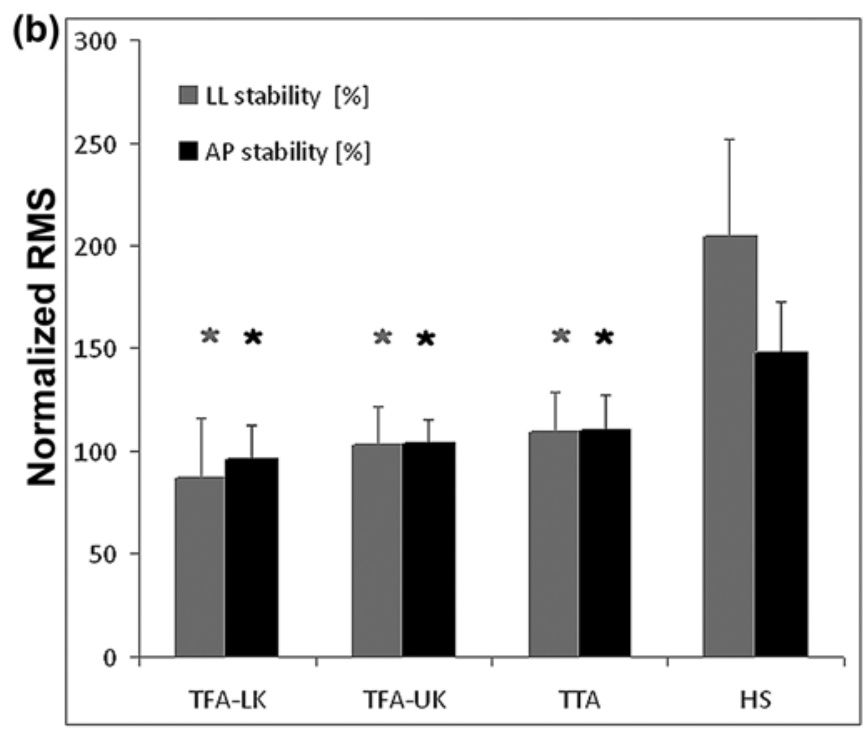

Subjects

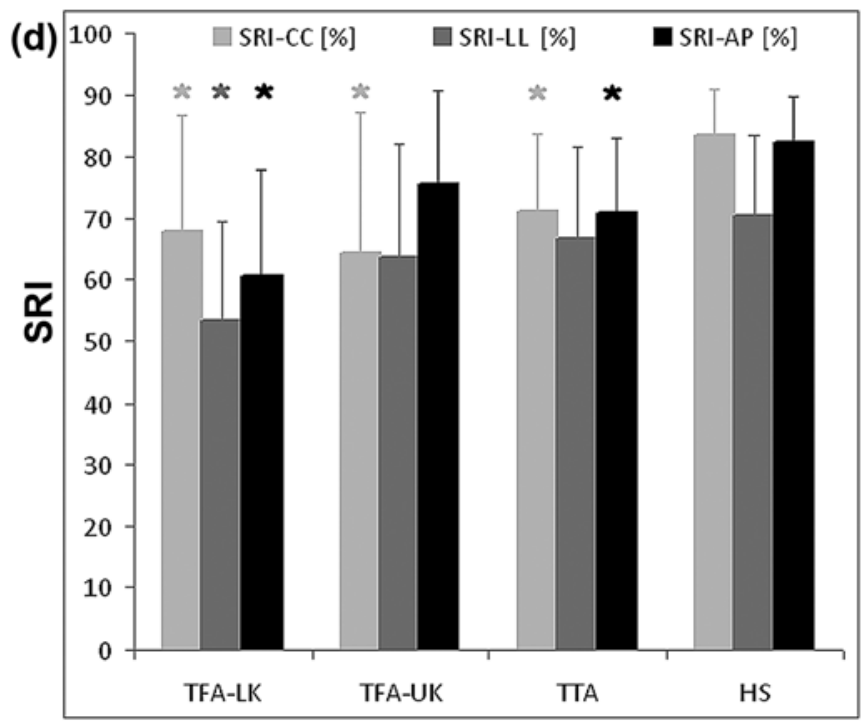

Subjects

Figure.

Biomechanical gait features for four groups of subjects. (a) Mean \pm standard deviation of walking speed (WS) and acceleration rootmean-square (RMS), (b) normalized RMS, (c) harmonic ratios (HRs), and (d) symmetry ratio index (SRI) of gait patterns along craniocaudal (CC), laterolateral (LL), and anteroposterior (AP) directions for subjects with transfemoral amputation with locked knee (TFA-LK), subjects with transfemoral amputation with unlocked knee (TFA-UK), subjects with transtibial amputation (TTA), and healthy subjects (HSs). "Indicates statistically significant difference for each parameter assessed between amputation group and HSs highlighted by post hoc comparisons.

features of gait, but not all of them at the same time. For example, the two subjects in our sample who walked faster than $1 \mathrm{~m} / \mathrm{s}$ optimized gait functioning but with low LL harmony. On the other hand, it is well known that speeds higher than the comfortable one decrease gait stability and harmony [13] and increase the risk of falls [38].

When we normalized accelerations using RMS-CC (and even when WS was used as a covariate variable) to 
compensate for the effects of speed, significant reduction was found for stability along the AP and LL axes, especially the latter, with respect to HSs. Thus, only after that normalization did we find a result similar to the one reported by Lamoth et al. for TFA subjects [1], i.e., a larger difference between subjects with amputation and controls in LL trunk accelerations than in AP trunk accelerations.

Furthermore, with respect to previous studies, this study also analyzed harmony of walking in subjects with amputation. Two recent studies have highlighted the importance of this feature of gait. A low value of the HR of trunk acceleration has been highlighted as a prognostic factor for the risk of falling in the elderly [38]. Moreover, harmony has recently been shown to be a fundamental feature of physiological human gait, with specific iterative proportions among repetitive gait phases related to the so-called golden ratio [39].

Our results showed very low values of HR for subjects with amputation: they were up to 90 percent lower than those of HSs for subjects with TFA walking with an LK prosthesis in the AP direction. These values were even lower than those observed in patients with stroke at dismissal from a rehabilitation hospital [24] and in children with cerebral palsy [12]. The biomechanical and sensory asymmetric changes that occur in subjects with amputation seemed to affect harmony of gait even more than neurological disorders. Conversely, stability (in terms of normalized RMS) was more impaired in the population with stroke [24] than in our sample of subjects with amputation. If there is a direct relationship between RMS and WS and increasing or decreasing gait velocity results in a corresponding increase or decrease in acceleration amplitude [24], the relationship between HR and speed seems more complicated.

Indeed, as shown by Menz et al., HR peaks at selfselected speeds and decreases at both slower and faster speeds [13]. This feature suggests changes in the spectrum content of acceleration signals at different speeds [35], probably involving aspects more related to motor control. Although all subjects involved in our study were asked to walk at their self-selected comfortable speed, we repeated our analysis on the HR also using WS as a covariate variable. We found that the main results did not change and that HR values remained statistically different among groups. Two cases of subjects with TTA walking fast with a high HR-AP, but with a low HR-LL, confirmed that the dependence of HR on WS was not strictly linear (like that of RMS). In any case, further studies are needed to clarify the role of speed on the spectrum content of upper-body accelerations.

In terms of gait symmetry, despite the different parameters used to compute the symmetry index, in subjects with TFA we found symmetry values between 50 and 75 percent and in HSs between 70 and 85 percent, thus not far from those found by Tura et al. [9], which were between 40 and 60 percent for subjects with amputation and 80 and 90 percent for HSs. A general trend from TFA-LK to TFA-UK to TTA subjects was found also for symmetry. However, AP symmetry was significantly different from that of HSs in TTA subjects but not in TFA-UK subjects. Again, subjects with TTA are probably able to walk faster, but lose in terms of symmetry and harmony of gait. Furthermore, TFA-UK subjects probably need to adopt a locomotor strategy to exploit the inertia of the prosthesis along the progressive direction, hence favoring AP symmetry despite a slight reduction in CC symmetry. Once these analyses were adjusted for WS, the main group effect on SRI lost statistical significance. There may be two reasons for this. The first was methodological and related to the choice to assess SRI as the ratio between two consecutive minima of acceleration signals, which are strictly linked to and affected by WS; thus, when the WS effect was removed, the SRI values were more similar among groups. The second is more general: most impaired subjects with amputation usually have asymmetric and slow gait; thus, considering speed as a covariate variable may reduce the effect of impairment on asymmetry.

Although symmetry was conceptually linked to harmony of gait, we observed some differences in terms of group effects between these two parameters. Perhaps this was because in our study, symmetry was evaluated by comparing the accelerations of the two limbs at foot strikes, whereas harmony took into account the accelerometric signal along the entire gait cycle. Moreover, the higher differences were noted along the LL axis. As reported previously, symmetry was evaluated using a step-by-step comparison (such as harmony) in the AP and CC axes, whereas in the LL axis, harmony was more related to stride cycle $[13,19]$.

Our study has some limits. We enrolled a larger sample of subjects with amputation with respect to previous studies investigating trunk accelerations during walking ( $N=10$ in Tura et al. [9], $N=8$ in Lamoth et al. [1]), but the division into three subgroups meant that there were only seven or eight subjects in each subgroup. This small 
subgroup size prevented us from further classifying subjects according to age, reason for amputation, or time since amputation (e.g., Hermodsson et al. demonstrated that postural standing sway was markedly different in patients with amputation of traumatic origin vs vascular origin [40] and that some gait features were different depending on the cause of amputation [41]).

Furthermore, we did not assess the possible stabilizing effect of using crutches or tetrapods, which could have influenced our results. For example, it has been shown that using supports has positive effects on WS and gait symmetry in patients with stroke [42-43]. This aspect has not been investigated in subjects with amputation, but in our study the only subject in the TFA-LK group who walked without aids showed higher stability in the AP direction (115.9\% vs $92.2 \%$ for other subjects in the same group), but he also showed reduced stability in the LL direction (74.4\% vs $88.6 \%$ ). Conversely, only one of the subjects with TTA used crutches and his values of RMS and HR in the LL direction were close to those of other subjects with TTA. Obviously, the clinical decision process took into account observational assessment of subject stability, which generated a confounding factor between stabilization due to crutches and better internal control. The risk of falling was much too high in our sample to ask subjects who needed crutches to walk without them, and this prevented us from designing a test-retest study with and without aids.

Another limit was that different prosthetic components were allowed in this study: we differentiated only between subjects with TFA using prostheses with LKs and UKs. Nevertheless, the present study is the first one on upper-body acceleration during walking in subjects with amputation upon dismissal from a rehabilitation hospital including TTA and TFA subjects and dividing subjects according to type of prosthesis used (i.e., UK or LK).

\section{CONCLUSIONS}

With respect to previous studies investigating upperbody stability during gait in younger and long-time prosthesis users, we found lower stability, harmony, and symmetry in a sample of subjects with amputation upon dismissal from a rehabilitation hospital. We also identified a clear trend depending on level of amputation and type of prosthesis for the stability, harmony, and symmetry evaluated on the sagittal plane. Accelerations in the
LL direction seemed more related to motor control than biomechanical issues. Rehabilitators should train subjects with amputation to progressively optimize gait features such as stability, efficiency, and functioning by making a safe choice about WS. Our study, together with previous ones $[1,9,27]$, also showed that the use of a single triaxial accelerometer (i.e., a low-cost, wearable, and easy-to-use device [44]) can provide useful quantitative and objective information about important gait features. The common walking tests performed in clinics on subjects with amputation, such as the $10 \mathrm{~m}$ walking test or the 6 min walking test [45], just measure speed of walking without providing information about the optimization of other important aspects of gait. Our study also showed that fast speed may not be a safe choice, because it may lead to less harmonic gait, exposing subjects to a higher risk of falling $[17,38]$.

In training people with amputation to use prostheses, researchers should focus interventions on the most impaired aspect of walking, i.e., harmony, a feature recently shown to be crucial for optimizing physiological gait [38-39].

\section{ACKNOWLEDGMENTS}

\section{Author Contributions:}

Study concept and design: M. Iosa, F. Paradisi.

Acquisition of data: F. Paradisi.

Analysis and interpretation of data: M. Iosa, F. Paradisi, S. Brunelli, A. S. Delussu, R. Pellegrini, D. Zenardi, S. Paolucci, M. Traballesi.

Drafting of manuscript: M. Iosa.

Critical revision of manuscript for important intellectual content:

M. Iosa, F. Paradisi, S. Brunelli, A. S. Delussu, R. Pellegrini,

D. Zenardi, S. Paolucci, M. Traballesi.

Drafting of manuscript: M. Iosa.

Statistical analysis: M. Iosa.

Obtained funding: S. Paolucci, M. Traballesi.

Administrative, technical, or material support: R. Pellegrini, D. Zenardi.

Study supervision: S. Paolucci, M. Traballesi.

Financial Disclosures: The authors have declared that no competing interests exist.

Funding/Support: This material was based on work supported by the Santa Lucia Foundation, Scientific Institute for Research, Hospitalization and Health Care.

Institutional Review: This study was approved by the ethical committee of the Santa Lucia Foundation, Scientific Institute for Research, Hospitalization and Health Care, and informed consent was obtained from each participant.

Participant Follow-Up: The authors do not plan to inform participants of the publication of this study. However, participants have been encouraged to check the study Web site for updated publications. 


\section{REFERENCES}

1. Lamoth CJ, Ainsworth E, Polomski W, Houdijk H. Variability and stability analysis of walking of transfemoral amputees. Med Eng Phys. 2010;32(9):1009-14.

[PMID:20685147]

http://dx.doi.org/10.1016/j.medengphy.2010.07.001

2. Ivanenko YP, Dominici N, Daprati E, Nico D, Cappellini G, Lacquaniti F. Locomotor body scheme. Hum Mov Sci. 2011;30(2):341-51. [PMID:21453667]

http://dx.doi.org/10.1016/j.humov.2010.04.001

3. Jaegers SM, Vos LD, Rispens P, Hof AL. The relationship between comfortable and most metabolically efficient walking speed in persons with unilateral above-knee amputation. Arch Phys Med Rehabil. 1993;74(5):521-25. [PMID:8489363]

http://dx.doi.org/10.1016/0003-9993(93)90117-S

4. Viton JM, Mouchnino L, Mille ML, Cincera M, Delarque A, Pedotti A, Bardot A, Massion J. Equilibrium and movement control strategies in trans-tibial amputees. Prosthet Orthot Int. 2000;24(2):108-16. [PMID:11061197] http://dx.doi.org/10.1080/03093640008726533

5. Chin T, Sawamura S, Fujita H, Ojima I, Oyabu H, Nagakura Y, Otsuka H, Nakagawa A. \%VO2max as an indicator of prosthetic rehabilitation outcome after dysvascular amputation. Prosthet Orthot Int. 2002;26(1):44-49. [PMID:12043925] http://dx.doi.org/10.1080/03093640208726620

6. Delussu AS, Brunelli S, Paradisi F, Iosa M, Pellegrini R, Zenardi D, Traballesi M. Assessment of the effects of carbon fiber and bionic foot during overground and treadmill walking in transtibial amputees. Gait Posture. 2013;38(4): 876-82. [PMID:23702342] http://dx.doi.org/10.1016/j.gaitpost.2013.04.009

7. Wezenberg D, de Haan A, Faber WX, Slootman HJ, van der Woude LH, Houdijk H. Peak oxygen consumption in older adults with a lower limb amputation. Arch Phys Med Rehabil. 2012;93(11):1924-29. [PMID:22684050] http://dx.doi.org/10.1016/j.apmr.2012.05.020

8. Mohanty RK, Lenka P, Equebal A, Kumar R. Comparison of energy cost in transtibial amputees using "prosthesis" and "crutches without prosthesis" for walking activities. Ann Phys Rehabil Med. 2012;55(4):252-62.

[PMID:22534430]

http://dx.doi.org/10.1016/j.rehab.2012.02.006

9. Tura A, Raggi M, Rocchi L, Cutti AG, Chiari L. Gait symmetry and regularity in transfemoral amputees assessed by trunk accelerations. J Neuroeng Rehabil. 2010;7:4.

[PMID:20085653]

http://dx.doi.org/10.1186/1743-0003-7-4

10. Cappozzo A. Low frequency self-generated vibration during ambulation in normal men. J Biomech. 1982;15(8):
599-609. [PMID:7142226]

http://dx.doi.org/10.1016/0021-9290(82)90071-9

11. Iosa M, Morelli D, Marro T, Paolucci S, Fusco A. Ability and stability of running and walking in children with cerebral palsy. Neuropediatrics. 2013;44(3):147-54.

[PMID:23487325]

http://dx.doi.org/10.1055/s-0033-1336016

12. Iosa M, Marro T, Paolucci S, Morelli D. Stability and harmony of gait in children with cerebral palsy. Res Dev Disabil. 2012;33(1):129-35. [PMID:22093657]

http://dx.doi.org/10.1016/j.ridd.2011.08.031

13. Menz HB, Lord SR, Fitzpatrick RC. Acceleration patterns of the head and pelvis when walking on level and irregular surfaces. Gait Posture. 2003;18(1):35-46.

[PMID:12855299]

http://dx.doi.org/10.1016/S0966-6362(02)00159-5

14. Dingwell JB, Marin LC. Kinematic variability and local dynamic stability of upper body motions when walking at different speeds. J Biomech. 2006;39(3):444-52.

[PMID:16389084]

http://dx.doi.org/10.1016/j.jbiomech.2004.12.014

15. Terrier P, Dériaz O. Kinematic variability, fractal dynamics and local dynamic stability of treadmill walking. J Neuroeng Rehabil. 2011;8:12. [PMID:21345241]

http://dx.doi.org/10.1186/1743-0003-8-12

16. Toebes MJ, Hoozemans MJ, Furrer R, Dekker J, van Dieën $\mathrm{JH}$. Local dynamic stability and variability of gait are associated with fall history in elderly subjects. Gait Posture. 2012;36(3):527-31. [PMID:22748312] http://dx.doi.org/10.1016/j.gaitpost.2012.05.016

17. Senden R, Savelberg HH, Grimm B, Heyligers IC, Meijer $\mathrm{K}$. Accelerometry-based gait analysis, an additional objective approach to screen subjects at risk for falling. Gait Posture. 2012;36(2):296-300. [PMID:22512847] http://dx.doi.org/10.1016/j.gaitpost.2012.03.015

18. Kavanagh JJ, Barrett RS, Morrison S. Upper body accelerations during walking in healthy young and elderly men. Gait Posture. 2004;20(3):291-98. [PMID:15531176] http://dx.doi.org/10.1016/j.gaitpost.2003.10.004

19. Mazzà C, Iosa M, Pecoraro F, Cappozzo A. Control of the upper body accelerations in young and elderly women during level walking. J Neuroeng Rehabil. 2008;5:30. [PMID:19014631] http://dx.doi.org/10.1186/1743-0003-5-30

20. Mazzà C, Iosa M, Picerno P, Cappozzo A. Gender differences in the control of the upper body accelerations during level walking. Gait Posture. 2009;29(2):300-303. [PMID:19013799] http://dx.doi.org/10.1016/j.gaitpost.2008.09.013

21. Mazzà C, Zok M, Cappozzo A. Head stabilization in children of both genders during level walking. Gait Posture. 
2010;31(4):429-32. [PMID:20163963]

http://dx.doi.org/10.1016/j.gaitpost.2010.01.012

22. Marigold DS, Patla AE. Age-related changes in gait for multi-surface terrain. Gait Posture. 2008;27(4):689-96. [PMID:17962018] http://dx.doi.org/10.1016/j.gaitpost.2007.09.005

23. Mizuike C, Ohgi S, Morita S. Analysis of stroke patient walking dynamics using a tri-axial accelerometer. Gait Posture. 2009;30(1):60-64. [PMID:19349181]

http://dx.doi.org/10.1016/j.gaitpost.2009.02.017

24. Iosa M, Fusco A, Morone G, Pratesi L, Coiro P, Venturiero V, De Angelis D, Bragoni M, Paolucci S. Assessment of upper-body dynamic stability during walking in patients with subacute stroke. J Rehabil Res Dev. 2012;49(3):439-50. [PMID:22773202] http://dx.doi.org/10.1682/JRRD.2011.03.0057

25. Lamoth CJ, Meijer OG, Wuisman PI, van Dieën JH, Levin MF, Beek PJ. Pelvis-thorax coordination in the transverse plane during walking in persons with nonspecific low back pain. Spine. 2002;27(4):E92-99. [PMID:11840116] http://dx.doi.org/10.1097/00007632-200202150-00016

26. Lamoth CJ, van Deudekom FJ, van Campen JP, Appels BA, de Vries OJ, Pijnappels M. Gait stability and variability measures show effects of impaired cognition and dual tasking in frail people. J Neuroeng Rehabil. 2011;8:2.

[PMID:21241487] http://dx.doi.org/10.1186/1743-0003-8-2

27. Tura A, Rocchi L, Raggi M, Cutti AG, Chiari L. Recommended number of strides for automatic assessment of gait symmetry and regularity in above-knee amputees by means of accelerometry and autocorrelation analysis. J Neuroeng Rehabil. 2012;9:11. [PMID:22316184] http://dx.doi.org/10.1186/1743-0003-9-11

28. Miller WC, Speechley M, Deathe B. The prevalence and risk factors of falling and fear of falling among lower extremity amputees. Arch Phys Med Rehabil. 2001;82(8): 1031-37. [PMID:11494181] http://dx.doi.org/10.1053/apmr.2001.24295

29. Mahoney FI, Barthel DW. Functional evaluation: The Barthel index. Md State Med J. 1965;14:61-65. [PMID:14258950]

30. Bohannon RW. Walking after stroke: Comfortable versus maximum safe speed. Int J Rehabil Res. 1992;15(3):246-48. [PMID:1428391] http://dx.doi.org/10.1097/00004356-199209000-00009

31. Zijlstra W, Hof AL. Assessment of spatio-temporal gait parameters from trunk accelerations during human walking. Gait Posture. 2003;18(2):1-10. [PMID:14654202] http://dx.doi.org/10.1016/S0966-6362(02)00190-X

32. Seliktar R, Mizrahi J. Some gait characteristics of belowknee amputees and their reflection on the ground reaction forces. Eng Med. 1986;15(1):27-34. [PMID:3699235]

http://dx.doi.org/10.1243/EMED JOUR 198601500902

33. Iosa M, Mazzà C, Pecoraro F, Aprile I, Ricci E, Cappozzo A. Control of the upper body movements during level walking in patients with facioscapulohumeral dystrophy. Gait Posture. 2010;31(1):68-72. [PMID:19782569]

http://dx.doi.org/10.1016/j.gaitpost.2009.08.247

34. Kolarova B, Janura M, Svoboda Z, Elfmark M. Limits of stability in persons with transtibial amputation with respect to prosthetic alignment alterations. Arch Phys Med Rehabil. 2013;94(11):2234-40. [PMID:23774381] http://dx.doi.org/10.1016/j.apmr.2013.05.019

35. Brach JS, McGurl D, Wert D, Vanswearingen JM, Perera S, Cham R, Studenski S. Validation of a measure of smoothness of walking. J Gerontol A Biol Sci Med Sci. 2011;66(1):136-41. [PMID:20923910] http://dx.doi.org/10.1093/gerona/glq170

36. Brunelli S, Averna T, Porcacchia P, Paolucci S, Di Meo F, Traballesi M. Functional status and factors influencing the rehabilitation outcome of people affected by above-knee amputation and hemiparesis. Arch Phys Med Rehabil. 2006;87(7):995-1000. [PMID:16813789]

http://dx.doi.org/10.1016/j.apmr.2006.04.004

37. Iosa M, Morone G, Fusco A, Pratesi L, Bragoni M, Coiro P, Multari M, Venturiero V, De Angelis D, Paolucci S. Effects of walking endurance reduction on gait stability in patients with stroke. Stroke Res Treat. 2012;2012:810415.

[PMID:21966598]

http://dx.doi.org/10.1155/2012/810415

38. Doi T, Hirata S, Ono R, Tsutsumimoto K, Misu S, Ando H. The harmonic ratio of trunk acceleration predicts falling among older people: Results of a 1-year prospective study. J Neuroeng Rehabil. 2013;10:7. [PMID:23356576] http://dx.doi.org/10.1186/1743-0003-10-7

39. Iosa M, Fusco A, Marchetti F, Morone G, Caltagirone C, Paolucci S, Peppe A. The golden ratio of gait harmony: repetitive proportions of repetitive gait phases. Biomed Res Int. 2013;2013:918642. [PMID:23862161] http://dx.doi.org/10.1155/2013/918642

40. Hermodsson Y, Ekdahl C, Persson BM, Roxendal G. Standing balance in trans-tibial amputees following vascular disease or trauma: A comparative study with healthy subjects. Prosthet Orthot Int. 1994;18(3):150-58. [PMID:7724348]

41. Hermodsson Y, Ekdahl C, Persson BM, Roxendal G. Gait in male trans-tibial amputees: A comparative study with healthy subjects in relation to walking speed. Prosthet Orthot Int. 1994;18(2):68-77. [PMID:7991363]

42. Beauchamp MK, Skrela M, Southmayd D, Trick J, Kessel MV, Brunton K, Inness E, Mcllroy WE. Immediate effects of cane use on gait symmetry in individuals with subacute stroke. Physiother Can. 2009;61(3):154-60. 
JRRD, Volume 51, Number 4, 2014

\section{[PMID:20514177]}

http://dx.doi.org/10.3138/physio.61.3.154

43. Hesse S, Jahnke MT, Schaffrin A, Lucke D, Reiter F, Konrad $\mathrm{M}$. Immediate effects of therapeutic facilitation on the gait of hemiparetic patients as compared with walking with and without a cane. Electroencephalogr Clin Neurophysiol. 1998;109(6):515-22. [PMID:10030684]

http://dx.doi.org/10.1016/S1388-2457(98)00033-9

44. Iosa M, Morone G, Fusco A, Bragoni M, Coiro P, Multari M, Venturiero V, De Angelis D, Pratesi L, Paolucci S. Seven capital devices for the future of stroke rehabilitation. Stroke Res Treat. 2012;2012:187965. [PMID:23304640] http://dx.doi.org/10.1155/2012/187965

45. Tekin L, Safaz Y, Göktepe AS, Yazýcýodlu K. Comparison of quality of life and functionality in patients with traumatic unilateral below knee amputation and salvage surgery. Prosthet Orthot Int. 2009;33(1):17-24. [PMID:19235062] http://dx.doi.org/10.1080/03093640802482542
Submitted for publication July 16, 2013. Accepted in revised form December 5, 2013.

This article and any supplementary material should be cited as follows:

Iosa M, Paradisi F, Brunelli S, Delussu AS, Pellegrini R, Zenardi D, Paolucci S, Traballesi M. Assessment of gait stability, harmony, and symmetry in subjects with lowerlimb amputation evaluated by trunk accelerations. J Rehabil Res Dev. 2014;51(4):623-34.

http://dx.doi.org/10.1682/JRRD.2013.07.0162

ResearcherID/ORCID: Marco Iosa, PhD: 0000-00032434-3887

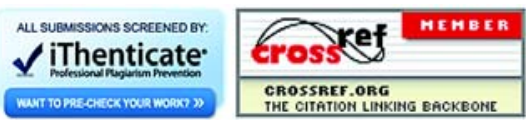

